\title{
TU/e emonown

\section{The influence of edge geometry on end-correction coefficients in micro perforated plates}

\section{Citation for published version (APA):}

Temiz, M. A., Lopez Arteaga, I., Efraimsson, G., Åbom, M., \& Hirschberg, A. (2015). The influence of edge geometry on end-correction coefficients in micro perforated plates. Journal of the Acoustical Society of America, 138(6), 3668-3677. https://doi.org/10.1121/1.4937748

DOI:

$10.1121 / 1.4937748$

Document status and date:

Published: 01/12/2015

\section{Document Version:}

Publisher's PDF, also known as Version of Record (includes final page, issue and volume numbers)

\section{Please check the document version of this publication:}

- A submitted manuscript is the version of the article upon submission and before peer-review. There can be important differences between the submitted version and the official published version of record. People interested in the research are advised to contact the author for the final version of the publication, or visit the $\mathrm{DOI}$ to the publisher's website.

- The final author version and the galley proof are versions of the publication after peer review.

- The final published version features the final layout of the paper including the volume, issue and page numbers.

Link to publication

\section{General rights}

Copyright and moral rights for the publications made accessible in the public portal are retained by the authors and/or other copyright owners and it is a condition of accessing publications that users recognise and abide by the legal requirements associated with these rights.

- Users may download and print one copy of any publication from the public portal for the purpose of private study or research.

- You may not further distribute the material or use it for any profit-making activity or commercial gain

- You may freely distribute the URL identifying the publication in the public portal.

If the publication is distributed under the terms of Article 25fa of the Dutch Copyright Act, indicated by the "Taverne" license above, please follow below link for the End User Agreement:

www.tue.nl/taverne

Take down policy

If you believe that this document breaches copyright please contact us at:

openaccess@tue.nl

providing details and we will investigate your claim. 


\title{
The influence of edge geometry on end-correction coefficients in micro perforated plates
}

\author{
Muttalip Aşkın Temiz ${ }^{a)}$ and Ines Lopez Arteaga \\ Department of Mechanical Engineering, Dynamics and Control, Eindhoven University of Technology, \\ P.O. Box 513, 5600 MB Eindhoven, The Netherlands \\ Gunilla Efraimsson \\ Department of Aeronautical and Vehicle Engineering, Centre for ECO2 Vehicle Design, \\ KTH Royal Institute of Technology, SE-100 44 Stockholm, Sweden \\ Mats Åbom \\ Department of Aeronautical and Vehicle Engineering, Linné Flow Centre, The Marcus Wallenberg Laboratory \\ for Sound and Vibration Research, KTH Royal Institute of Technology, SE-100 44 Stockholm, Sweden \\ Avraham Hirschberg \\ Department of Applied Physics, Gas Dynamics and Aero-Acoustics, Eindhoven University of Technology, \\ P.O. Box 513, 5600 MB Eindhoven, The Netherlands
}

(Received 17 March 2015; revised 22 October 2015; accepted 23 November 2015; published online 17 December 2015)

\begin{abstract}
Global expressions are proposed for end-correction coefficients in micro perforated plates (MPPs) using non-dimensional parameters. MPPs are sound absorbers with small perforation diameters such that the Stokes boundary layers fill up almost the entire perforation. Sound absorption does not only occur within the perforation, but also takes place just outside of it. The latter contribution plus the outside inertia effect on the transfer impedance of the MPP are referred to as end-corrections. In order to determine them, an analytical solution employing the very thin Stokes layer assumption has been derived. However, this assumption requires empirical coefficients in the end-corrections for accurate results. To explore the effects of various parameters a numerical model is used. This model is verified with open-end reflection coefficient measurements. The most prominent result from this study is that compared to plate thickness, the ratio of perforation diameter to Stokes layer thickness (Shear number) and edge geometry affect the end-correction coefficients more significantly. The effect of plate thickness can be neglected for practical purposes, therefore, expressions for the end-corrections in terms of Shear number and edge geometry are provided. The relative error of these expressions is $<3 \%$ compared to the numerical results. (C) 2015 Acoustical Society of America.
\end{abstract}

[http://dx.doi.org/10.1121/1.4937748]

[FCS]

Pages: $3668-3677$

\section{INTRODUCTION}

Micro perforated plates (MPPs) are plates with perforations whose diameters are on the order of a millimeter and with a low porosity, i.e., $\phi=\mathcal{O}(1 \%) .{ }^{1}$ Due to the small diameter size, the oscillating viscous boundary layers, i.e., Stokes layers, cover the majority of the perforation as illustrated in Fig. 1.

MPPs are identified as efficient absorbers by Maa. ${ }^{1}$ Maa combines the oscillating viscous flow in a capillary tube solution from Crandall, ${ }^{2}$ which is a simplified version of the visco-thermal derivation of Kirchhoff, ${ }^{3}$ with the endcorrections of Ingard. ${ }^{4}$ This way, $\mathrm{Maa}^{1}$ derives a transfer impedance expression for a single perforation. Nevertheless, Ingard ${ }^{4}$ bases his end-correction coefficient derivation on very thin Stokes layer assumption. Thus, this model does not represent the acoustic behaviour of MPPs for Stokes

\footnotetext{
a)Electronic mail: m.a.temiz@tue.nl
}

boundary layers as thick as the perforation radius, i.e., at low frequencies where the acoustic wavelength is large. It is an unrealistic assumption because it completely neglects the edge effect of the perforation geometry.

Consequently, the analytical model of $\mathrm{Maa}^{1}$ requires empirical coefficients to match the experimental results. Especially, the coefficient for the resistive end-correction varies between 2 and 4 in the literature and this has been associated with edge geometry by Allam and Åbom. ${ }^{5}$ On the other hand, the theoretical limit value for the reactive end-correction coefficient is reasonably accurate for most applications.

To solve this deficiency, especially with the resistive end-correction coefficient, Bolton and $\mathrm{Kim}^{6}$ have developed a numerical model in 2D-axisymmetric (cylindrical) coordinates. With this model they simulate viscous, incompressible, oscillating flow in the time domain. Bolton and $\mathrm{Kim}^{6}$ include the end effects of the perforation by using upstream and downstream channels with a fixed length of $1 \mathrm{~mm}$. They have run simulations for 21 different combinations of plate thickness 


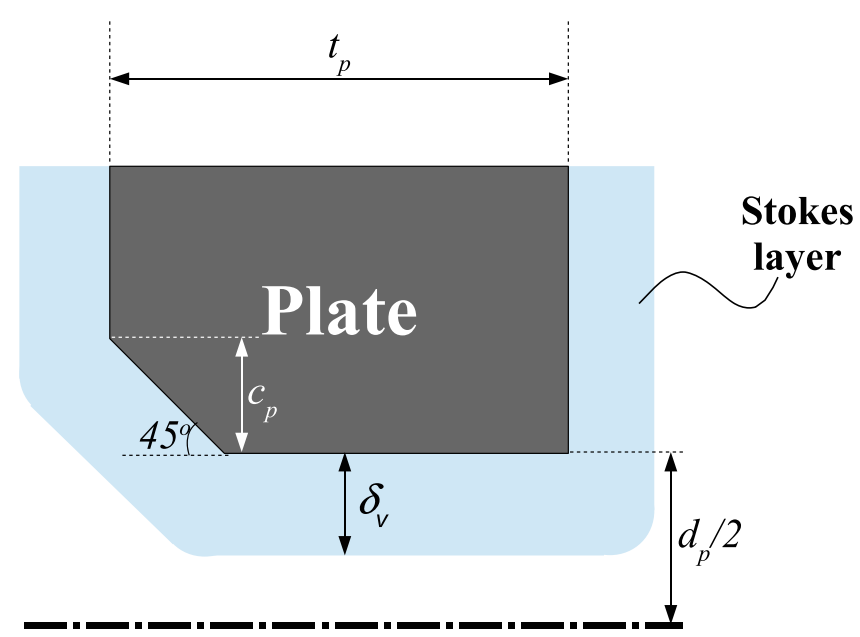

FIG. 1. (Color online) Representation of the Stokes layer $\left(\delta_{v}=\sqrt{\mu /\left(\rho_{0} \omega\right)}\right)$ within a single perforation of an MPP in 2D-axisymmetrical geometry. The parameters defining perforation diameter $\left(d_{p}\right)$, plate thickness $\left(t_{p}\right)$, and chamfer length $\left(c_{p}\right)$ are also shown.

$\left(t_{p}\right)$, perforation diameter $\left(d_{p}\right)$, and porosity $(\phi)$ parameters. Bolton and $\mathrm{Kim}^{6}$ proposed an expression for the resistive endcorrection coefficient in the dimensions of $\left(\mathrm{Hz}^{-0.5}\right)$.

Furthermore, using Bolton and Kim's CFD approach, ${ }^{6}$ Herdtle et $a l^{7}$ have computeed the end-corrections for tapered perforations.

In another recent study by Carbajo et al., ${ }^{8}$ a method similar to that of Bolton and $\mathrm{Kim}^{6}$ is used to study the interaction between perforations. Although the two works mentioned above propose a valuable methodology, there is a need for a more generalized definition of the end-correction coefficients and experimental validation of the results. Furthermore, none of the studies discussed above considers the influence of the perforation edges on the acoustic performance of the MPP.

In this paper, both the resistive and reactive end-correction coefficients are evaluated by means of an axisymmetrical, incompressible flow model in the frequency domain and validated with experiments, also including the influence of the shape of the perforation edges. Although our approach is analogous to that of Bolton and $\mathrm{Kim},{ }^{6}$ our results are significantly different in the following aspects. First, we use nondimensional parameters to express end-correction coefficients so that the results are generalized and useful for the design of MPPs with circular perforations. Second, we solve linearized Navier-Stokes equations numerically in the frequency domain. Moreover, we make sure that the acoustic transfer impedance values are calculated independent of the inlet and outlet channel length. Finally, we investigate the effect of the edge shape geometry on the end-correction coefficients. In other words, our aim is to provide a consistent base for the calculation of the transfer impedance in MPPs with circular holes in the linear regime.

On the other hand, we limit our study to certain aspects. First of all, we only focus on low perforation rates $(\phi=\mathcal{O}(1 \%))$ so that we can ignore hydrodynamic interaction between perforations. ${ }^{1,5,6}$ Second, although slit shaped MPPs appear to be quite promising, ${ }^{5}$ here, we concentrate on circular perforation geometries so that they can be represented in 2D axisymmetrical geometry in numerical model. Finally, we carry out measurements with a single perforation diameter for all samples.

\section{THEORETICAL BACKGROUND}

The transfer impedance of an MPP is defined as

$$
Z_{t}=\frac{\Delta \hat{P}}{\phi \hat{U}_{p}}
$$

where $\Delta \hat{P}$ is the plane wave pressure difference across the plate, $\phi$ is the porosity, and $\hat{U}_{p}$ is the volume flow rate divided by the perforation area. From experimental or simulated data, plane wave pressure is obtained on each side of the plate by extrapolating a plane wave model up to the surface of the plate. When $\hat{U}_{p}$ is multiplied with $\phi$, we get plane wave normal velocity before (or after) the plate. Please note that the circumflex accent $\left(^{\wedge}\right)$ indicates complex quantity throughout the paper.

For MPPs with circular perforations, the transfer impedance with a finite plate thickness is modeled by $\mathrm{Maa}^{1}$ as follows:

$$
\begin{aligned}
Z_{t}= & j \omega t_{p} \rho_{0}\left[1-\frac{2}{\operatorname{Sh} \sqrt{-j}} \frac{J_{1}(\operatorname{Sh} \sqrt{-j})}{J_{0}(\operatorname{Sh} \sqrt{-j})}\right]^{-1}+2 \alpha R_{S} \\
& +j \delta \omega \rho_{0} \frac{d_{p}}{2}
\end{aligned}
$$

using the $\exp (j \omega t)$ convention.

The first term on the right-hand side of Eq. (2) defines the oscillating fluid flow within the perforation where $\omega=2 \pi f$ is the radial frequency, $\rho_{0}$ is the density of air $\left(1.184 \mathrm{~kg} / \mathrm{m}^{3}\right.$ at $20^{\circ} \mathrm{C}, 1.205 \mathrm{~kg} / \mathrm{m}^{3}$ at $\left.25^{\circ} \mathrm{C}\right), j$ is the imaginary number $\sqrt{-1}, J_{n}$ is the Bessel function of first kind of order $n$, and $\mathrm{Sh}$ is the Shear number, which is defined ${ }^{9}$ as $\mathrm{Sh}=d_{p} \sqrt{\omega \rho_{0} /(4 \mu)}$, where $\mu$ is the dynamic viscosity of air $\left(1.82 \times 10^{-5} \mathrm{~kg} / \mathrm{ms}\right.$ at $20^{\circ} \mathrm{C}, 1.84 \times 10^{-5} \mathrm{~kg} / \mathrm{ms}$ at $\left.25^{\circ} \mathrm{C}\right)$.

The second term on the right-hand side of Eq. (2) is the resistive end-correction expression, and the last term is the reactive (inertial) end-correction expression. $R_{S}$ is the surface resistance on one side of the plate, which is calculated by $R_{S}=0.5 \sqrt{2 \mu \rho_{0} \omega}$. Moreover, the non-dimensional resistive and the reactive end-correction coefficients are denoted by $\alpha$ and $\delta$ in Eq. (2). The end effects become very important in plates with normalized thickness, $t^{*}=t_{p} / d_{p}$, on the order of unity.

Even though $\mathrm{Maa}^{1}$ has proposed his analytical model for the sharp-edge perforation case, the presence of endcorrection coefficients in the model provides the flexibility to include different edge types.

We start our study with the sharp-edge case and, then, extend this study for various types and combinations for chamfered edges. We expect the behaviour of the chamfered edges to be similar to those with rounded edges. Chamfers are preferred to roundings due to manufacturing accuracy. 

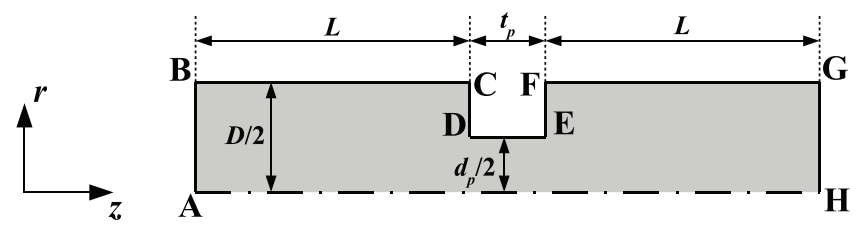

FIG. 2. Computational domain of a single perforation. $|\mathrm{AB}|$, harmonic velocity inlet; $|\mathrm{BC}|$ and $|\mathrm{FG}|$, slip wall; $|\mathrm{CD}|,|\mathrm{DE}|$, and $|\mathrm{EF}|$, no-slip wall; $|\mathrm{GH}|$, viscous-free, zero-pressure outlet; and $|\mathrm{AH}|$, radial symmetry axis.

\section{NUMERICAL SET-UP}

The numerical part of the study contains a model tailored to a single perforation. We assume a viscous, incompressible flow in $2 \mathrm{D}$ axisymmetric coordinates. To compare the results with Maa's linear model and extend it further, we keep our calculations in the linear regime also. As a result, in the model, we solve the following linearized incompressible Navier-Stokes equations in the frequency domain:

$$
\begin{aligned}
& \frac{\partial \hat{u}_{r}}{\partial r}+\frac{\hat{u}_{r}}{r}+\frac{\partial \hat{u}_{z}}{\partial z}=0, \\
& j \rho_{0} \omega \hat{u}_{r}+\frac{\partial \hat{p}}{\partial r}-\mu\left(\frac{\partial^{2} \hat{u}_{r}}{\partial r^{2}}+\frac{1}{r} \frac{\partial \hat{u}_{r}}{\partial r}+\frac{\partial^{2} \hat{u}_{r}}{\partial z^{2}}\right)=0, \\
& j \rho_{0} \omega \hat{u}_{z}+\frac{\partial \hat{p}}{\partial z}-\mu\left(\frac{\partial^{2} \hat{u}_{z}}{\partial r^{2}}+\frac{1}{r} \frac{\partial \hat{u}_{z}}{\partial r}+\frac{\partial^{2} \hat{u}_{z}}{\partial z^{2}}\right)=0,
\end{aligned}
$$

where $r$ and $z$ represents the radial and axial axes components, respectively; $\rho_{0}$ represents the base flow density; $\hat{u}$ and $\hat{p}$ represent acoustic velocity and pressure, respectively. In COMSOL Multiphysics ${ }^{\circledR}$ (Stockholm, Sweden), Eq. (3) is discretized using finite elements in the Coefficient Form Partial Differential Equation module of the program. We used quadratic elements in our simulations. Since the flow is laminar, no turbulence model was needed in the simulations.

A schematic drawing of the computational domain and the boundary conditions used are presented in Fig. 2.
As can be seen from Fig. 2, the geometry covers both inner and outer regions of the perforation. The outer region is the upstream/downstream channel and its diameter is taken as $D=d_{p} / \sqrt{\phi}$. Doing that, we make sure the effect of the perforation is negligible at the inlet and outlet boundaries. During the course of the simulations we saw that an increase of $33 \%$ in the channel length results with a change of $<0.001 \%$ in the pressure amplitude, which also indicates that a long enough computational domain was used. A typical pressure distribution obtained by solving linearized incompressible Navier-Stokes equations is shown in Fig. 3.

We calculate the transfer impedance dividing the acoustic pressure difference between two sides of the perforation by the volume flux per perforation area, as stated in Eq. (1). The relevant acoustic pressure at one side of the perforation is obtained by linear extrapolation, assuming an incompressible uniform flow, from the inlet (or the outlet) boundary of the computational domain to the surface of the perforation. ${ }^{10}$ In our incompressible model, the pressure difference $(\Delta \hat{P})$ is therefore given by

$$
\Delta \hat{P}=\hat{P}_{u}-\hat{P}_{d}-j 2 L \rho_{0} \omega \hat{U},
$$

where $\hat{P}_{u}=\hat{p}\left(z_{A B}\right)$ and $\hat{P}_{d}=\hat{p}\left(z_{G H}\right)$ are the upstream and the downstream pressures at the inlet and the outlet of the numerical domain, respectively. Furthermore, $\hat{U}$ is the imposed uniform inlet velocity of the model and $L$ is the length of the upstream and downstream sections. The correction given in Eq. (4) ensures that $\Delta \hat{P}$ is independent of the upstream/downstream channel length.

In order to avoid the need for resolving sharp edges, we employ fillets with small radius, $r_{f}$, at the edge points. The simulations are repeated for different fillet radius to perforation diameter values, $r_{f} / d_{p}$, such as $6.25 \times 10^{-3}, 3.13 \times 10^{-3}$, and $1.56 \times 10^{-3}$. Observing the change is linear, we extrapolate $Z_{t}$ to the $r_{f}=0$ case and approximate the limit value as accurately as possible.

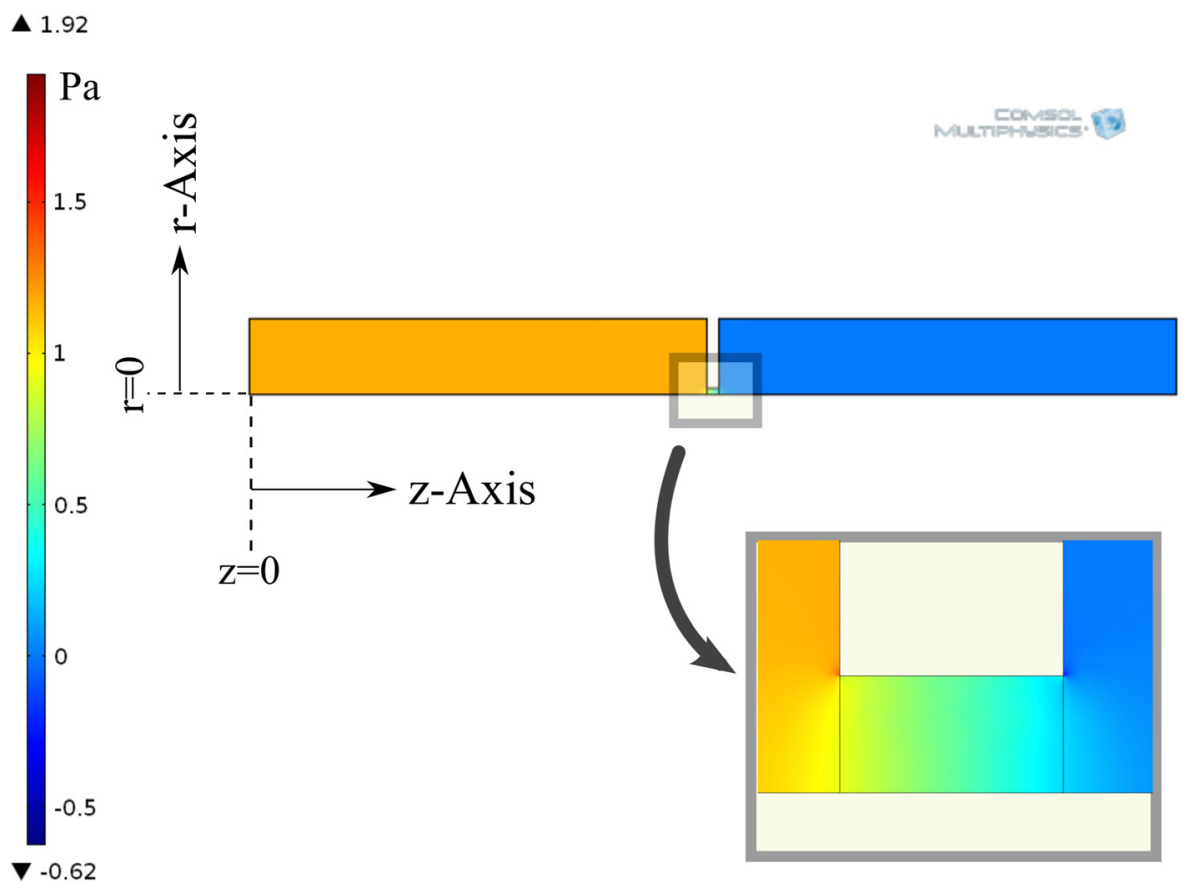

FIG. 3. (Color online) A typical pressure amplitude distribution around a sharp-edged perforation. 


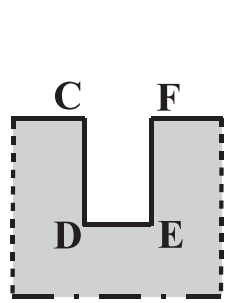

(I)

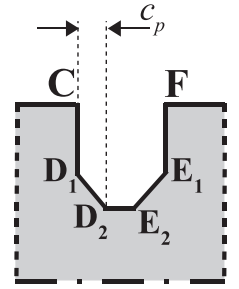

(II)

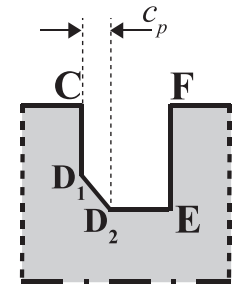

(III)

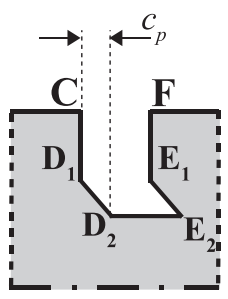

(IV)

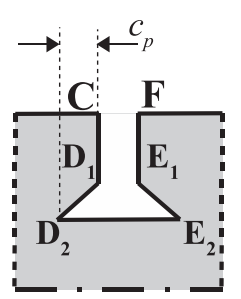

(V)
FIG. 4. Edge geometries investigated in this study: (I) sharp-edge, (II) both ends chamfered, (III) one end chamfered, (IV) punched hole geometry, (V) inverse-chamfered.

Using this approach, not only sharp-edged perforations but also other types of hole geometries are investigated in our study. The schematic representations of these geometries are shown in Fig. 4.

In all the cases shown in Fig. 4, the sharp corners are taken care of with the method mentioned above to avoid numerical singularity.

With COMSOL Multiphysics ${ }^{\circledR,}$ s built-in mesh generation tool, we build a mesh with non-constant distribution to minimize the number of grid points. This results in a combination of triangular and rectangular mesh types. Although triangular mesh dominates the domain, in the vicinity of the boundaries with no-slip boundary condition $(B C)$, rectangular mesh type is present. An example for this distribution is shown in Fig. 5.

A mesh study resulted in that, for the mesh used, the difference in the value of $Z_{t}$ is $<0.02 \%$ compared to the successive finer grid level.

\section{EXPERIMENTAL VERIFICATION}

The verification of our numerical model is done by comparing four different cases with experimental results. In all these cases, we use samples with a single perforation as in the numerical model. Their properties are given in Table I and their photo is provided in Fig. 6.

The samples introduced in Table I are placed at the end of a $1 \mathrm{~m}$ long impedance tube. The tube is made of aluminium with an inner diameter of $50 \mathrm{~mm}$ and a wall thickness of $10 \mathrm{~mm}$. A photo and a schematic drawing of the system are given in Fig. 7.

We use NI PCIe-6361 X-Series (National Instruments, Austin, TX) data acquisition card with 16 analog input and 2 analog output channels. We generate and record signals using LabView $^{\circledR}$. We use one output channel for the loudspeaker and six input channels for the microphones. The type of the microphones is BSWA MPA416 (BSWA Tech, Beijing, China) with a sensitivity of $50.45 \mathrm{mV} / \mathrm{Pa}$. They are equally distributed by a distance of $175 \mathrm{~mm}$. This setup employs the algorithm described in Fig. 8 to perform reflection coefficient measurements.

For the calibration of the microphones, we perform a one-time measurement before the others. Using a calibration mount specially designed for this purpose, we place all the microphones at the same distance from the loudspeaker and the closed end tube termination. The idea is that: for each frequency step, every microphone should read the same

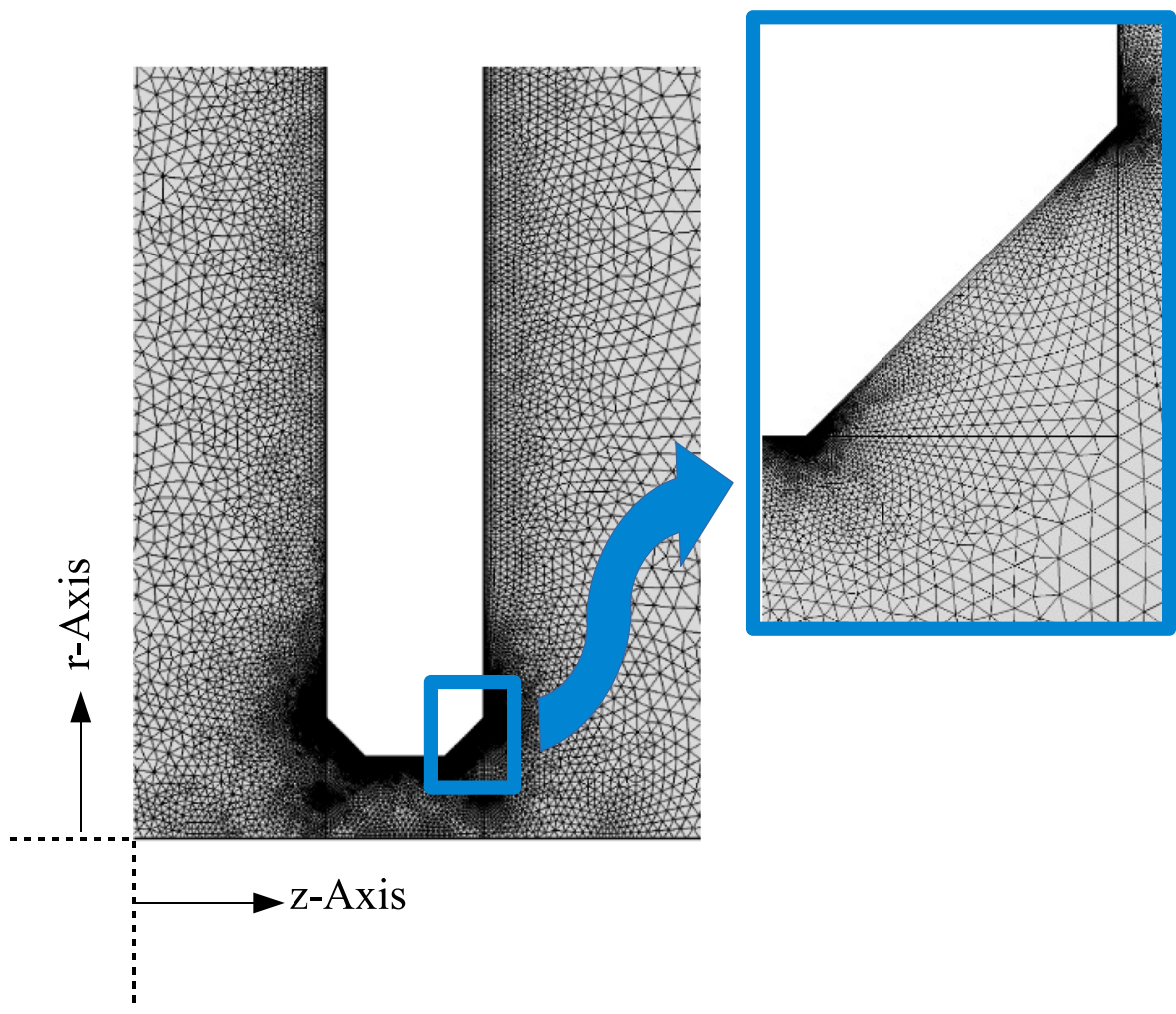

FIG. 5. (Color online) An example of how non-constant distribution of the mesh looks like around the both-sideschamfered perforation. Note that the mesh is finer in the vicinity of the edges. 
TABLE I. Sample specifications.

\begin{tabular}{|c|c|c|c|c|c|}
\hline Sample Name & $d_{p}(\mathrm{~mm})$ & $t_{p}(\mathrm{~mm})$ & $\phi$ & Edge Type & $c_{p}(\mathrm{~mm})$ \\
\hline Sample I & $4.20 \pm 0.05$ & $4.00 \pm 0.01$ & $0.71 \%$ & Sharp & N/A \\
\hline Sample II & $4.20 \pm 0.05$ & $4.00 \pm 0.01$ & $0.71 \%$ & One-side-chamfered & $0.35 \pm 0.05$ \\
\hline Sample III & $4.20 \pm 0.05$ & $4.00 \pm 0.01$ & $0.71 \%$ & Both-sides-chamfered & $1.00 \pm 0.05$ \\
\hline Sample IV & $4.20 \pm 0.05$ & $4.00 \pm 0.01$ & $0.71 \%$ & Punched & $1.00 \pm 0.05$ \\
\hline
\end{tabular}

complex pressure value according to the plane wave assumption. As a result, we select one of the microphones as the reference and force other microphones to have the same value for the same frequency. We do this by calculating the calibration coefficients for each frequency setup. This procedure is a relative calibration technique. However, since we perform reflection coefficient measurements, an absolute calibration is not necessary. Please note that, in this technique, the arbitrarily selected reference microphone should remain the same throughout all the measurements. Finally, the microphones are relatively calibrated for the frequency range $[100,700] \mathrm{Hz}$. Please note that, although the tube allows us to carry on measurements approximately up $3.4 \mathrm{kHz}$; considering the sample dimensions, the viscous effects are expected to be small enough to be neglected for $f>700 \mathrm{kHz}$ $(\mathrm{Sh}>35)$. Moreover, above this frequency, the influence of the finite compliance of the microphones becomes significant. ${ }^{11}$ We did not correct for this effect.

In the measurements of MPPs, after the pressure data from the microphones are saved, we calculate the corresponding reflection coefficient for each frequency step by another script we built. This script omits the first and last $3 \mathrm{~s}$ from the measurements to avoid transient effects and uses the calibration coefficients obtained before.

Both of the scripts calculating the calibration and reflection coefficients employ a lock-in method. ${ }^{12}$ This method correlates the input signal to the output to calculate the measurements in the Fourier domain. The advantage of this method over FFT (or DFT) is that it ensures there is no spectral leakage.

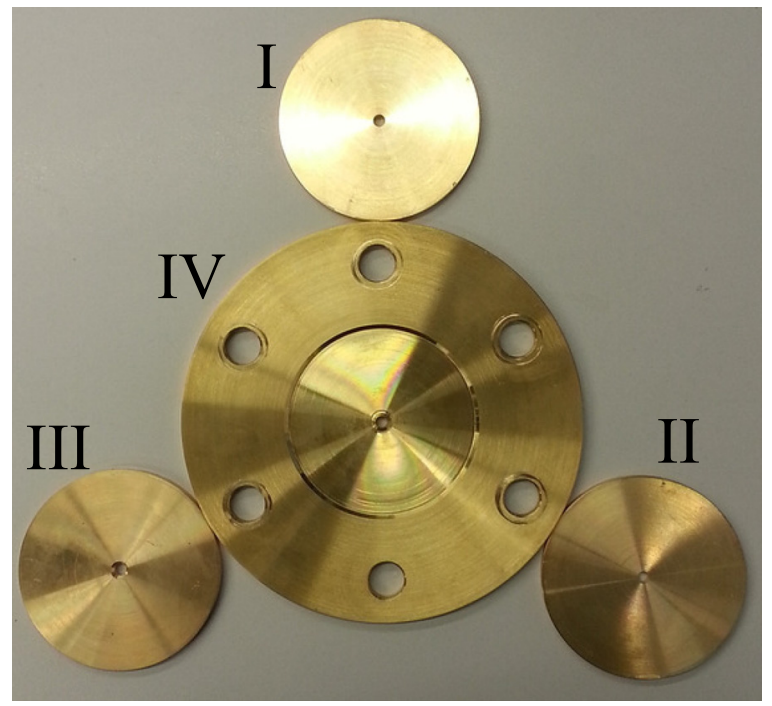

FIG. 6. (Color online) MPP samples used in the impedance tube to verify the numerical model. Samples I, II, and III require a sample holder, while sample IV has it built in.
The calculation of the reflection coefficient is based on the plane wave assumption. In other words, within the tube, all the points at position $z$ are assumed to have the same complex pressure amplitude $\hat{p}(z)$ and this can be decomposed into right, $\hat{p}_{+}$, and left, $\hat{p}_{-}$, traveling pressure waves such as

$$
\hat{p}(z)=\hat{p}_{+} \exp \left(-j k_{c} z\right)+\hat{p}_{-} \exp \left(j k_{c} z\right),
$$

where $k_{c}$ is the complex wave number taking visco-thermal effects into account and is described by Peters et al. ${ }^{9}$ as follows:

$$
\begin{aligned}
k_{c}= & \frac{\omega}{c_{0}}\left[1+\frac{1-j}{\sqrt{2} \operatorname{Sh}}\left(1+\frac{\gamma-1}{\operatorname{Pr}^{0.5}}\right)\right] \\
& -\frac{\omega}{c_{0}}\left[\frac{j}{\operatorname{Sh}^{2}}\left(1+\frac{\gamma-1}{\operatorname{Pr}^{0.5}}-\frac{1}{2} \gamma \frac{\gamma-1}{\operatorname{Pr}}\right)\right],
\end{aligned}
$$

where $\operatorname{Pr}$ is the Prandtl number and $\gamma$ is the heat capacity ratio. In our calculations, we omit the term with $\mathrm{Sh}^{2}$ since its value does not exceed $2 \%$ of the first order term in Eq. (6).
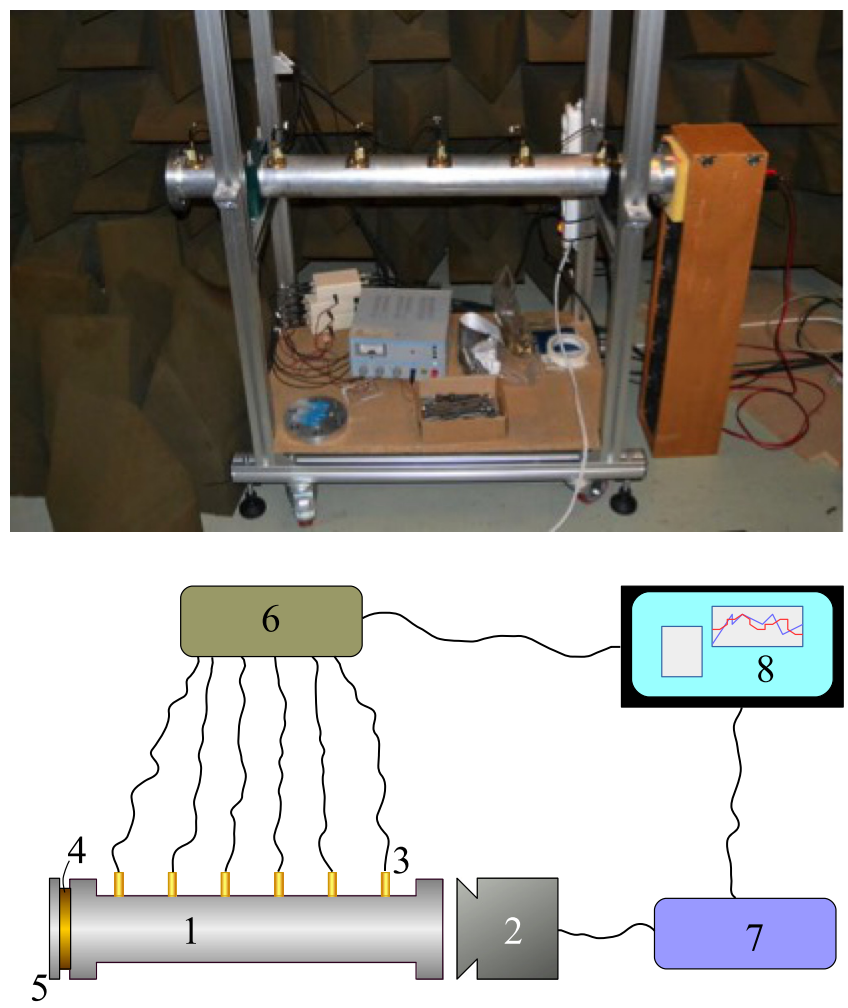

FIG. 7. (Color online) The setup used for verification of the numerical model. (a) The photo of the setup; (b) schematic drawing of the setup: (1) the impedance tube, (2) loudspeaker, (3) microphones, (4) MPP sample, (5) (hollow) sample holder, (6) microphone amplifier, (7) loudspeaker amplifier, (8) analyzer and computer. 


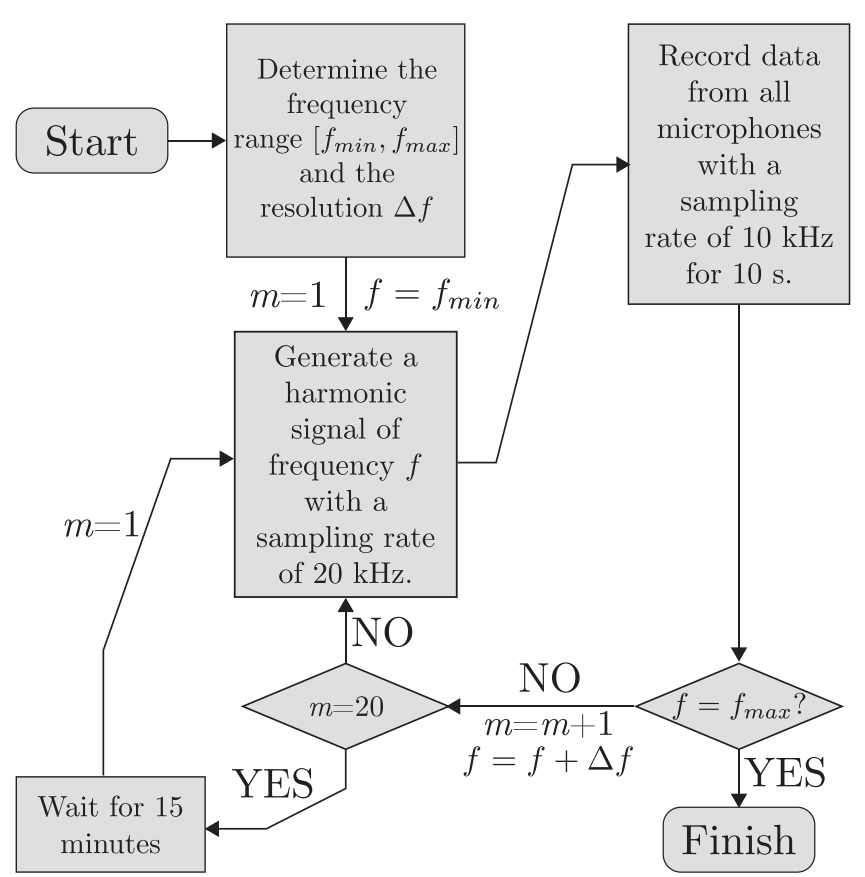

FIG. 8. Measurement algorithm.

Introducing this complex wave number into the method of least square fit for six microphones by Jang and $\mathrm{Ih},{ }^{13}$ the plane wave decomposition is obtained and the reflection coefficient can be expressed as

$$
R=\frac{\hat{p}_{-} \exp \left(j k_{c} z\right)}{\hat{p}_{+} \exp \left(-j k_{c} z\right)} .
$$

In Fig. 8, one can see a 15-min delay between two successive 20-step measurements. The reason for this is to restore the uniform temperature in the tube. This is due to the fact that measurements are affected by the change in the speed of sound, $c_{0}$. For 20 frequency steps, we have 40 wave amplitudes as unknown, plus $c_{0}$ as an additional unknown. The signals of the 6 microphones provide a set of 120 equations for those 41 unknowns, which is solved by the least square method proposed by Aurégan and Leroux. ${ }^{14}$ For the completely closed-end case, the deviation of the measured reflection coefficient from the theoretical value $R=1.000$ is $<0.3 \%$.

We measure the transfer impedance by the following procedure:

(i) Measure the open end reflection coefficient of the open impedance tube without the sample plate, $R_{O E}$, and calculate the radiation impedance, $Z_{R}$, using $Z_{R}=\rho_{0} c_{0}\left(1+R_{O E}\right) /\left(1-R_{O E}\right)$.

(ii) Place the sample plate to the end, measure the reflection coefficient of sample-loaded end, $R_{P}$, and calculate $Z_{P}=\rho_{0} c_{0}\left(1+R_{P}\right) /\left(1-R_{P}\right)$.

(iii) Obtain the transfer impedance of the plate, $Z_{t}$, by subtracting the radiation impedance from the sampleloaded end impedance: $Z_{t}=Z_{P}-Z_{R}$.

The samples are attached in between the impedance tube and the hollow sample holder (see Fig. 7), whose inner diameter is the same as the tube and length is 1.5 times the diameter. Since tube terminations in both sample-loaded and open-end (without the sample) cases are identical and the surroundings are the same, we expect the radiation impedance values to be the same. Besides, one should note that since $\phi \sim \mathcal{O}(1 \%)$ for the samples, $Z_{R}$ is expected to be much lower than $Z_{P}$ and the possible error is negligible in $Z_{t}=Z_{R}-Z_{P}$.

Being aware of the non-linearity issues in the MPP measurements, we employ an empirical procedure to avoid such effects. We decrease the excitation amplitude gradually at the lowest frequency of interest. When two successive measurements give the same reflection coefficient value, we carry out the measurements for the frequency range with that particular amplitude. This is based on the definition of Strouhal number, $\mathrm{Sr}=\phi \omega d_{p} /|\hat{U}|$, by Ingard and Ising. ${ }^{15}$ According to this definition if $\mathrm{Sr}>1$, we do not observe non-linear effects in the MPPs and $\mathrm{Sr}$ increases with increasing frequency. We furthermore verified that $\mathrm{Sr}$ remains larger than unity for all our measurements.

Comparison between the numerical model and the experiments is done in terms of non-dimensional end-correction coefficients $\alpha$ and $\delta$. We calculate these coefficients by rearranging Eq. (2)

$$
\begin{gathered}
\alpha=\frac{\left(\Re\left\{Z_{t}\right\}-\Re\left\{Z_{t}\right\}_{t h}\right) \phi}{2 R_{S}}, \\
\delta=\frac{\left(\Im\left\{Z_{t}\right\}-\Im\left\{Z_{t}\right\}_{t h}\right) \phi}{\rho_{0} \omega d_{p} / 2},
\end{gathered}
$$

where subscript th represents the word theoretical and this corresponds to the transfer impedance calculated by means of the theory by Crandall. ${ }^{2}$ In other words, the theoretical transfer impedance of a perforation is calculated by Eq. (2) without the end-corrections $(\alpha=\delta=0)$. His model takes into account only the inside of the perforation of length $t_{\mathrm{eff}}$. This effective thickness can be calculated as $t_{\text {eff }}=t_{p}-n c_{p}$ where $c_{p}$ is the chamfered length and the coefficient $n$ is the edge type factor whose values for different edge types are listed in Table II.

When employing Eq. (8) with the values of $Z_{t}$ determined from measurements, one obtains experimental values for $\alpha$ and $\delta$. Similarly, in order to obtain numerical endcorrection coefficients, $Z_{t}$ calculated by simulations should be used.

For the samples introduced in Table I, the comparison between numerical and experimental end-correction coefficients is shown in Fig. 9.

TABLE II. Factor $n$ for different edge geometries.

\begin{tabular}{lr}
\hline \hline Edge type & $n$ \\
\hline Sharp & 0 \\
One-side-chamfered & 1 \\
Both-sides-chamfered & 2 \\
One-side-inverse-chamfered & -1 \\
Both-sides-inverse-chamfered & -2 \\
Punched & 0 \\
\hline \hline
\end{tabular}



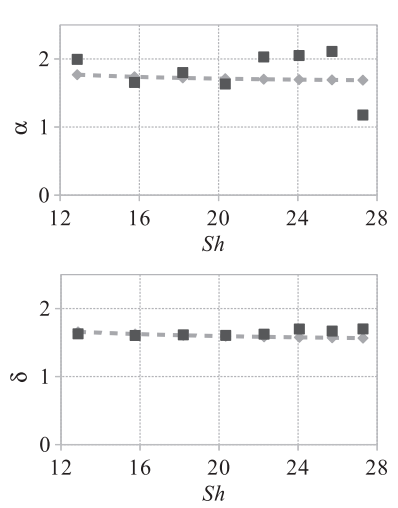

(a)
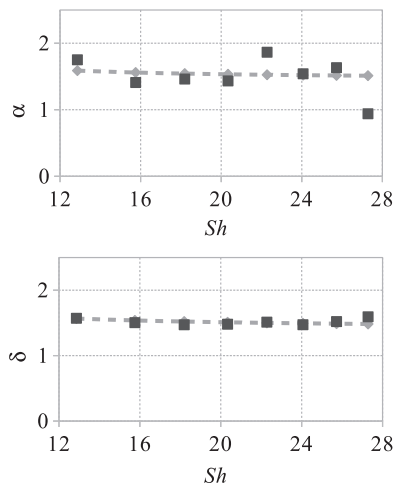

(b)
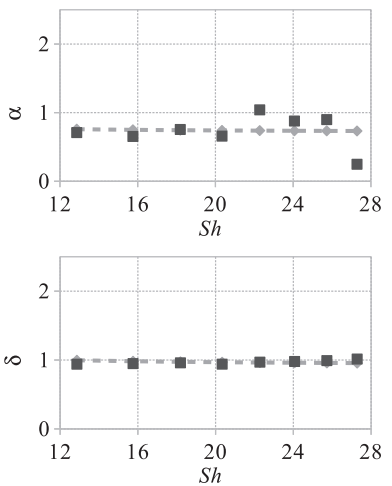

(c)
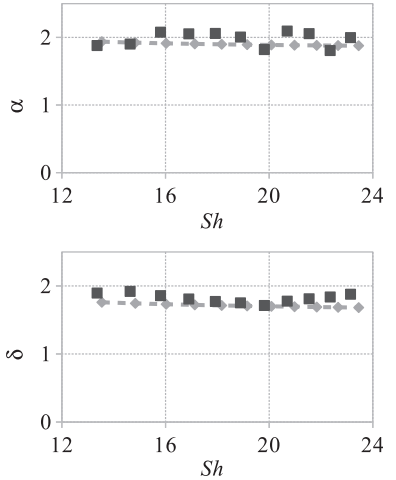

(d)

FIG. 9. Comparison of the resistive and reactive end-correction coefficients of samples with different edge geometries:

experimental, $->-$, numerical results.

In this paper, we concentrate on samples whose perforation diameter is rather larger than a typical MPP for the verification of our numerical model. This results in a higher Sh number in the frequency of interest, i.e., $\mathrm{Sh}>10$. The numerical model presented here has been already validated in the low Sh number region, $1<\mathrm{Sh}<14$, for sharp edges. ${ }^{16}$ In this previous study, imperfections in the perforation geometry of some of the samples are observed with holes that seem to have a triangular rather than circular shape. We concluded that, to ensure a high accuracy of the hole geometry and edge shape, larger hole diameter and thickness values should be used. As a result, the samples described in Table I are produced and tested.

Although the open-end impedance measurement is easy to apply, it has a disadvantage where the reflection coefficient value approaches unity [recall $Z_{R}=\rho_{0} c_{0}\left(1+R_{O E}\right)$ / $\left(1-R_{O E}\right)$ ]. A very small disturbance when $|R| \approx 1$ can lead to large errors in transfer impedance. Thus, in all measurements we have large uncertainty above $450-500 \mathrm{~Hz}$ and we, therefore, present results up to $\mathrm{Sh} \approx 27$. It can be concluded from the results in Fig. 9 that the numerically determined end-correction coefficients are in good agreement with the experimental values for the range of Sh numbers considered. Therefore, the numerical model proposed is validated and will be used in Sec. V to calculate the end-correction coefficients for perforations with different types of edges.

\section{RESULTS}

After experimental verification, we use our numerical model to broaden the study for $1<\mathrm{Sh}<35$. Doing so, we aim to cover the important $\mathrm{Sh}$ number region, $1<\mathrm{Sh}<10$ for the MPPs according to $\mathrm{Maa}^{1}$ and extend it to theoretical limits where the end-correction coefficients are comparable with our results. We divide our study in three main classes according to the perforation edge geometry.

\section{A. Perforations with sharp edges}

The properties of the numerical cases designed to cover the Sh number range of interest are presented in Table III.

With the use of Eq. (8), we calculate $\alpha$ and $\delta$ from the simulations for each case. To observe the effect of the thickness of the Stokes layer on these coefficients, we present the results from all cases in two graphs: $\alpha$ vs Sh and $\delta$ vs Sh, which can be seen in Fig. 10.

From Fig. 10, we observe a strong dependence on Sh for both $\alpha$ and $\delta$. Assuming that Sh is the only parameter defining the end-correction coefficients in sharp-edge perforations, we propose a practical expression for computing $\alpha$ and $\delta$ for $1<\mathrm{Sh}<35$ and $\mathrm{Sr}>1$ as follows:

$$
\begin{aligned}
& \alpha_{s}=5.08 \mathrm{Sh}^{-1.45}+1.70 \\
& \delta_{s}=0.97 \exp (-0.20 \mathrm{Sh})+1.54
\end{aligned}
$$

where subscript " $s$ " represents the perforations with the sharp edge geometry.

The expressions given in Eq. (9) consist of fit formulas from 411 data points with a quality of $R_{\alpha}^{2}=0.9995$ and $R_{\delta}^{2}=0.9960$. The curves calculated using Eq. (9) are compared to numerical data in Fig. 10.

We extend our investigation on sharp-edged perforations with the non-dimensional plate thickness, $t^{*}=t_{p} / d_{p}$. This time, we perform a surface fit with two independent parameters to include thickness effect in $\alpha$ and $\delta$. The updated expressions with the wall thickness for the endcorrection coefficients for the perforations with sharp edges become

$$
\begin{aligned}
& \alpha_{s}=5.08 \mathrm{Sh}^{-1.45}+1.70-0.002 / t^{*} \\
& \delta_{s}=0.97 \exp (-0.20 \mathrm{Sh})+1.54-0.003 / t^{*}
\end{aligned}
$$

The new fits given in Eq. (10) have a marginally better quality, $R_{\alpha}^{2}=0.9995$ and $R_{\delta}^{2}=0.9961$, so we conclude that $\alpha$ and $\delta$ do not significantly depend on $t^{*}$ for $t^{*} \geq 0.5$.

TABLE III. Properties of the sharp-edge cases investigated numerically.

\begin{tabular}{lccc}
\hline \hline & $d_{p}(\mathrm{~mm})$ & $t_{p}(\mathrm{~mm})$ & $\phi$ \\
\hline Case 1 & 0.3 & 1.0 & $0.77 \%$ \\
Case 2 & 0.8 & 0.4 & $0.74 \%$ \\
Case 3 & 0.8 & 1.0 & $0.74 \%$ \\
Case 4 & 0.8 & 8.0 & $0.74 \%$ \\
Case 5 & 1.6 & 1.6 & $0.72 \%$ \\
Case 6 & 4.2 & 4.0 & $0.71 \%$ \\
\hline \hline
\end{tabular}




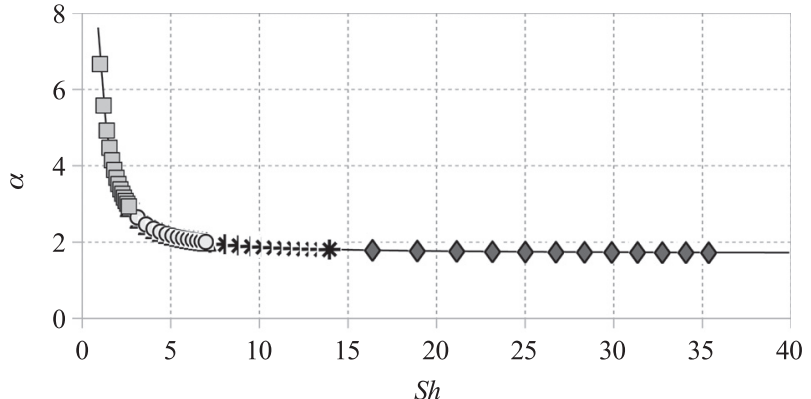

(a)

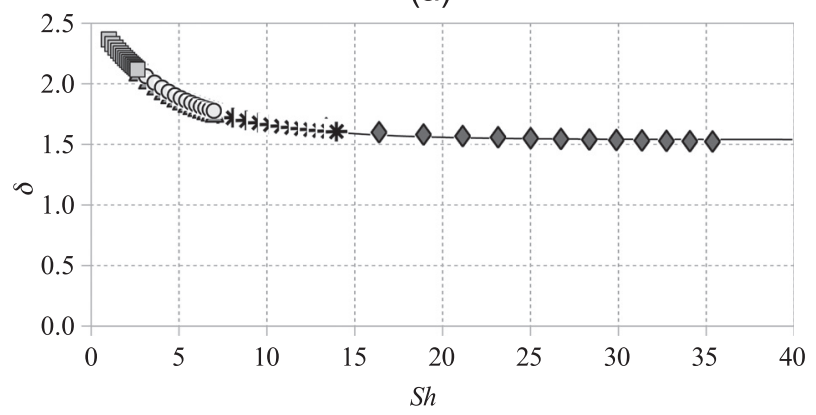

(b)

FIG. 10. End-correction coefficients plotted as functions of Sh: , case 1; , case $2 ; \boldsymbol{\Lambda}$, case $3 ; \nabla$, case $4 ; \boldsymbol{*}$, case $5 ; \boldsymbol{\diamond}$, case 6 ; and - , proposed fit formula.

\section{B. Perforations with chamfered edges}

We classify chamfers in two types depending on if it reduces or increases the effective plate thickness, $t_{\mathrm{eff}}$. The chamfered edge with $45^{\circ}$ angle reduces $t_{\text {eff }}$ and is defined as normal, whereas the one with $135^{\circ}$ angle increases $t_{\text {eff }}$ and is defined as inverse chamfer. Recall that $t_{\mathrm{eff}}=t_{p}-n c_{p}$, where $n$ can be obtained from Table II. While calculating the theoretical transfer impedance by Kirchhoff, ${ }^{3}$ effective thickness should be used.

Even though they have different profiles at the perforation edges, the definition of the chamfer length, $c_{p}$, and the non-dimensional chamfer length, $c^{*}=c_{p} / d_{p}$, are still the same for both normal and inverted chamfers. The properties of the numerical cases designed for investigating chamfers can be seen in Table IV. The limit $c^{*}=0$ is the case of sharp-edge and should be taken into account to relate the results with the previous part of the study. For this reason, case 6 is included in both normal and inverse chamfer types.

For this part of the study, we consider four different cases. These cases include the smallest and largest nondimensional chamfer length limits, i.e., $c^{*}=0$ and $c^{*}=t^{*} / 2$,

TABLE IV. Properties of the chamfered-edge cases investigated numerically.

\begin{tabular}{lccccc}
\hline \hline & $d_{p}[\mathrm{~mm}]$ & $t_{p}[\mathrm{~mm}]$ & $\phi$ & $c_{p}[\mathrm{~mm}]$ & Type \\
\hline Case 7 & 4.2 & 4.0 & $0.71 \%$ & 0.35 & Normal \\
Case 8 & 4.2 & 4.0 & $0.71 \%$ & 1.0 & Normal \\
Case 9 & 4.2 & 4.0 & $0.71 \%$ & 2.0 & Normal \\
Case 10 & 4.2 & 4.0 & $0.71 \%$ & 0.50 & Inverse \\
Case 11 & 4.2 & 4.0 & $0.71 \%$ & 1.0 & Inverse \\
Case 12 & 4.2 & 4.0 & $0.71 \%$ & 2.0 & Inverse \\
\hline \hline
\end{tabular}

respectively. The properties of these numerical cases can be seen in Table IV.

Including the sharp edge geometry in both chamfer types, we have 244 data points for each coefficient in both types. For perforations with chamfered edges, the best surface representing the distribution of the points for $\alpha$ and $\delta$ are in $13<\mathrm{Sh}<35$ and $\mathrm{Sr}>1$.

$$
\begin{aligned}
\alpha_{c}= & 5.08 \mathrm{Sh}^{-1.45}+1.70+1.18 c^{* 1.74} \mathrm{Sh}^{-0.26}, \\
\delta_{c}= & 0.97 \exp (-0.20 \mathrm{Sh})+1.54 \\
& +0.97 c^{* 0.56} \exp (-0.01 \mathrm{Sh}),
\end{aligned}
$$

where the subscript " $c$ " represents the perforations with the chamfered edge geometry.

The quality of these fits can be quantified by $R_{\alpha}^{2}=0.9808$ and $R_{\delta}^{2}=0.9945$.

The same study on perforations with inverse-chamfered edges results with the following $\alpha$ and $\delta$ fits in $13<\mathrm{Sh}<35$ and $\mathrm{Sr}>1$ as

$$
\begin{aligned}
\alpha_{i c}= & 5.08 \mathrm{Sh}^{-1.45}+1.70+0.08 c^{* 0.17} \mathrm{Sh}^{0.36}, \\
\delta_{i c}= & 0.97 \exp (-0.20 \mathrm{Sh})+1.54 \\
& -0.17 c^{* 0.41} \exp (0.02 \mathrm{Sh}),
\end{aligned}
$$

where the subscript "ic" denotes the inverse-chamfered edge geometry.

For these fits, we calculate $R_{\alpha}^{2}=0.9986$ and $R_{\delta}^{2}=0.9883$.

\section{Combinations of edge geometries}

Neither sharp nor both-sides-chamfered edge geometries are easy to manufacture in mass production of the MPPs. Hence, we consider two geometries that can be used as practical approximations. These are one-side chamfered and punched hole geometries, which can be seen in Fig. 4.

In this part of the study, we run simulations for perforations with smaller diameters to cover lower Sh number region. The properties of the cases we simulated are listed in Table V.

\section{One-side-chamfered}

This geometry is considered for the cases where the perforations are opened with drills when supported by an

TABLE V. Properties of the numerical cases to verify the proposed fit. Cases $\mathrm{C} 1$ to $\mathrm{C} 4$ represent one-sided-chamfered edges where Cases P1 to P4 represent punched hole geometry. $\phi=0.71 \%$ for all cases.

\begin{tabular}{lccc}
\hline \hline & $d_{p}(\mathrm{~mm})$ & $t_{p}(\mathrm{~mm})$ & $c_{p}(\mathrm{~mm})$ \\
\hline Case C1 & 0.3 & 1.0 & 0.025 \\
Case C2 & 0.9 & 1.0 & 0.075 \\
Case C3 & 1.5 & 1.0 & 0.125 \\
Case C4 & 4.2 & 4.0 & 0.350 \\
Case P1 & 4.2 & 1.0 & 0.071 \\
Case P2 & 4.2 & 1.0 & 0.214 \\
Case P3 & 4.2 & 1.0 & 0.357 \\
Case P4 & 4.2 & 4.0 & 1.00 \\
\hline \hline
\end{tabular}


additional material from behind. One side of the perforation has the chamfer geometry where the other end is sharp. Referring to the linearity, we propose the end-corrections can be a combination of both cases mentioned as

$$
\begin{aligned}
& \alpha=\left(\alpha_{s}+\alpha_{c}\right) / 2, \\
& \delta=\left(\delta_{s}+\delta_{c}\right) / 2,
\end{aligned}
$$

where subscripts " $s$ " and " $c$ " denote end-corrections for sharp-edges and chamfered edges, respectively, which is calculated from Eqs. (9) and (11). We assume, these expressions hold for the lower $\mathrm{Sh}$ region as well since the governing physics is the same and we use non-dimensional numbers. To check that assumption, we select $c^{*}=0.083$ as in sample II, and compare the results of this linear combination with numerical simulations in terms of relative percent error, $\epsilon_{r}$. We calculate it for $\alpha$ as follows: $\epsilon_{r}=100 \mid \alpha_{\text {num }}$ $-\alpha_{\text {fit }} \mid / \alpha_{\text {num }}$. Replacing $\alpha$ with $\delta$, one obtains the same error definition for the reactive end-correction coefficient. These error plots are provided in Fig. 11.

\section{Punched hole}

The idea behind investigating this geometry is to approximate the perforations opened by punching the plate. This geometry consists of a chamfered edge and an inversechamfered edge.

Similar to the one-side-chamfered geometry, the endcorrection coefficients of this one can be calculated as

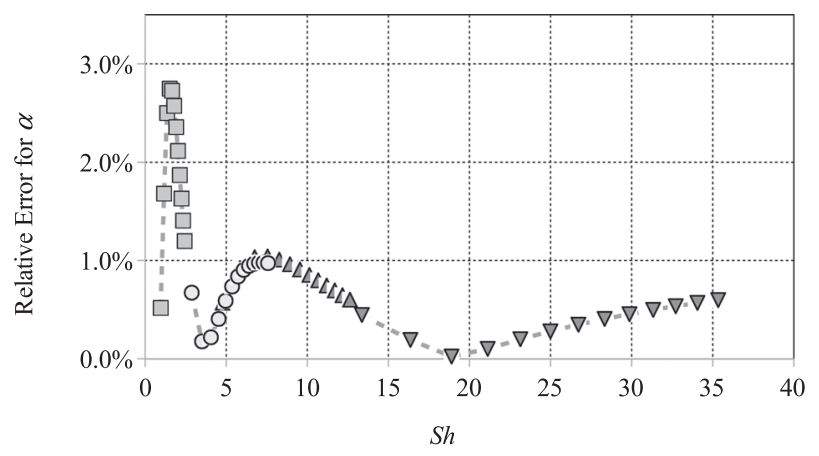

(a)

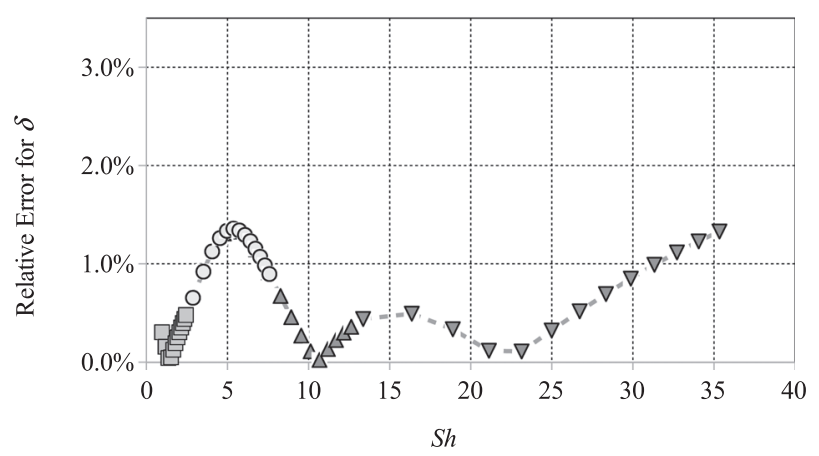

(b)

FIG. 11. The relative percent error of the end-correction coefficients obtained by the proposed fit wrt numerical simulations in one-side-chamfered edge profile. $-\quad-$, case $\mathrm{C} 1 ;-(-$, case $\mathrm{C} 2 ;-\triangle \ldots$, case $\mathrm{C} 3$; and $-\nabla=$, case C4.

$$
\begin{aligned}
& \alpha=\left(\alpha_{c}+\alpha_{i c}\right) / 2, \\
& \delta=\left(\delta_{c}+\delta_{i c}\right) / 2,
\end{aligned}
$$

where subscript "ic" stands for inverse-chamfered and can be calculated using Eq. (12). The relative percent error between the numerical results and the proposed fit is shown in Fig. 12.

Please recall that one-sided-chamfered edge is composed of sharp edge and (normal) chamfered edge types. Similarly, punched hole geometry is composed of (normal) chamfered edge and inverse edge types. Hence, verifying the linear combination of these edge types with numerical results is a compact verification of all the fits we propose. From Figs. 11 and 12, we see that even for low Sh number region, the fits and the numerical results are in accordance within $<3 \%$ in the Sh number region of interest. Hence, the assumption for the lower Sh number region holds and the fits we propose can be used for calculating end-correction coefficients of MPPs for $\mathrm{Sr}>1$.

\section{CONCLUDING REMARKS}

This study proposes expressions for dimensionless endcorrection coefficients, $\alpha$ and $\delta$ in MPPs based on numerical analysis, whose results are verified by experiments. Using the numerical model we built, different edge geometries such as sharp, chamfered, inverse chamfered edges, and their linear combinations are investigated.

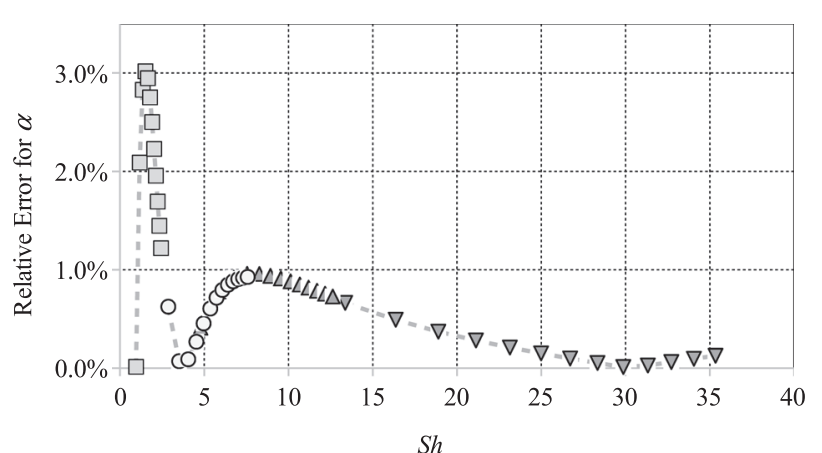

(a)

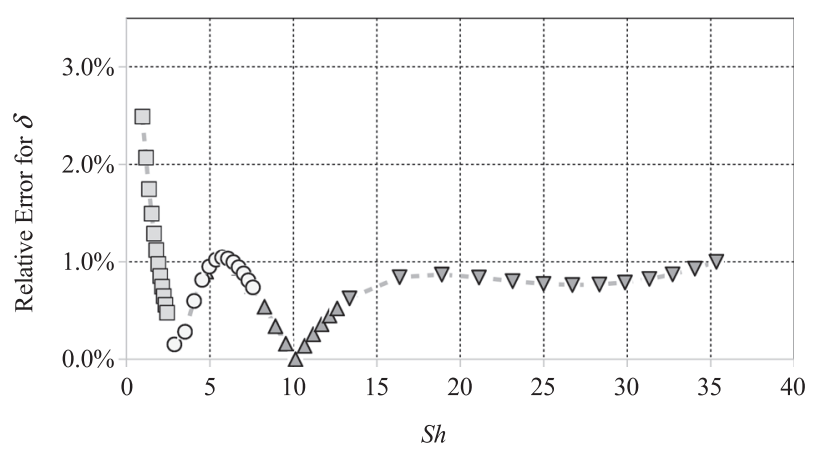

(b)

FIG. 12. The relative percent error of the end-correction coefficients obtained by the proposed fit wrt numerical simulations in punched hole geometry. $-\square-$, case $\mathrm{P} 1 ;-\mathrm{O}-$, case $\mathrm{P} 2 ;-\triangle-$, case $\mathrm{P} 3$; and $-\nabla=$, case P4. 
We measure the transfer impedance with the open-end method. Yet, when the amplitude of the reflection coefficient of the sample is close to 1 , the method becomes prone to errors. For this reason, the measurements with the MPP samples could go up to $450-500 \mathrm{~Hz}$. This corresponds to $\mathrm{Sh} \approx 27$ for samples $d_{p}=4.2 \mathrm{~mm}$. If studying with higher $\mathrm{Sh}$ is required, one should employ another experimental technique.

The numerical results show that the plate thickness has negligible effect on end-correction coefficients. We also conclude that $\alpha$ and $\delta$ strongly depend on Sh number and the edge geometry. These arguments have been tested in a large variety of non-dimensional thickness range such as $0.5<t^{*}<10$.

Sharp-edge profile is an important comparison case with the theory. It is reported in the literature that $\alpha$ can be taken as either 2 or $4 .^{5}$ Our results show that $\alpha$ value can be out of this interval depending on the Shear number. Moreover, with increasing Sh number, we observe an asymptotic approach in end-correction coefficients. These are 1.70 for $\alpha$ and 1.54 for $\delta$. Experiments with the samples also support this statement. Please recall Ingard ${ }^{4}$ proposed $\alpha$ to be 2 empirically; our result, $\alpha=1.70$, is close to this. Although Morse and Ingard ${ }^{17}$ proposes a purely analytical solution for $\alpha$, it does not show the asymptotic behaviour that we see in the experiments. On the other hand, both Morse and Ingard ${ }^{17}$ and Pierce $^{18}$ agree on the theoretical limit for $\delta=1.57$ in very thin plates. This value is comparable with our findings, $\delta=1.54$.

The chamfered-edge geometry increases $\alpha$ and $\delta$ compared to sharp-edge geometry. Yet, the overall transfer impedance value decreases in presence of chamfers. This is due to the fact that the viscous friction is dominant in the narrow part of the perforations, which is defined by $t_{\mathrm{eff}}$ in this study, and chamfers reduce this effective plate thickness. On the other hand, inverse-chamfered edges increase $t_{\text {eff }}$, resulting in a higher transfer impedance compared to sharpedges. In inverse-chamfers, the fluid particles must follow a streamline making a $135^{\circ}$ turn. This increases the resistance of the edge but makes it harder for fluid particles to oscillate in and out of the perforation. As a result, compared to sharpedge geometry, $\alpha$ increases but $\delta$ decreases in inversechamfered edges.

Since the entire investigation is carried out in linear regime, we combine end-correction coefficients for sharpedge, chamfered-edge, and inverse-chamfered-edge geometries linearly to obtain $\alpha$ and $\delta$ for one-side-chamfered edge and punched hole geometries. Although the fit is obtained from data in the region $13<\mathrm{Sh}<35$, the end-correction coefficients obtained with the proposed expressions are in good agreement with the numerical results even for the region $1<\mathrm{Sh}<13$. Moreover, the expressions proposed for chamfered geometries are designed to reduce into expressions for sharp-edge profiles when $c^{*}=0$ is selected.
Consequently, the expressions we offer are global and the error between them and the numerical results are $<3 \%$ for $1<\mathrm{Sh}<35$.

\section{ACKNOWLEDGMENTS}

The presented work is part of the Marie Curie Initial Training Network thermo-acoustic and aero-acoustic nonlinearities in green combustors with orifice structures (TANGO). We gratefully acknowledge the financial support from the European Commission under call FP7-PEOPLEITN-2012. Thanks to Fanny Depaix, Ronald Meijers, and Constant Hak for the pioneer work in the experiments.

${ }^{1}$ D.-Y. Maa, "Potential of microperforated panel absorber," J. Acoust. Soc. Am. 104(5), 2861-2866 (1998).

${ }^{2}$ I. B. Crandall, Theory of Vibrating Systems and Sound (van Nostrand Company, New York, 1926), pp. 229-237.

${ }^{3}$ G. Kirchhoff, "Über den Einfluss der Wärmeleitung in einem Gase auf die Schallbewegung" ("About the influence of heat conduction on the sound propagation in a gas"), Ann. Phys. Chem. 210(6), 177-193 (1868).

${ }^{4} \mathrm{~K}$. Ingard, "On the theory and design of acoustic resonators," J. Acoust. Soc. Am. 25(6), 1037-1061 (1953).

${ }^{5} \mathrm{~S}$. Allam and M. Ảbom, "A new type of muffler based on microperforated tubes," J. Vib. Acoust. 113(3), 031005 (2011).

${ }^{6}$ J. S. Bolton and N. Kim, "Use of CFD to calculate the dynamic resistive end correction for microperforated materials," Acoust. Aust. 38, 134-139 (2010).

${ }^{7}$ T. Herdtle, J. S. Bolton, N. N. Kim, J. H. Alexander, and R. W. Gerdes, "Transfer impedance of microperforated materials with tapered holes," J. Acoust. Soc. Am. 134(6), 4752-4762 (2013).

${ }^{8}$ J. Carbajo, J. Ramis, L. Godinho, P. Amado-Mendes, and J. Alba, “A finite element model of perforated panel absorbers including viscothermal effects," Appl. Acoust. 90, 1-8 (2015).

${ }^{9}$ M. C. A. M. Peters, A. Hirschberg, A. J. Reijnen, and A. P. J. Wijnands, "Damping and reflection coefficient measurements for an open pipe at low Mach and low Helmholtz numbers," J. Fluid. Mech. 256, 499-534 (1993).

${ }^{10} \mathrm{~T}$. Herdtle and J. S. Bolton, "Effect of thermal losses and fluid-structure interaction on the transfer impedance of microperforated films," in Proceedings of NOISE-CON2014, Fort Lauderdale, FL (2014), pp. 1-12.

${ }^{11} \mathrm{~L}$. Peerlings, "Methods and techniques for precise and accurate in-duct aero-acoustic measurements: Application to the area expansion," Licentiate thesis, KTH Royal Institute of Technology, Stockholm, Sweden, 2015, pp. 25-30.

${ }^{12}$ J. H. Scofield, "Frequency-domain description of a lock-in amplifier," Am. J. Phys. 62, 129-133 (1994).

${ }^{13} \mathrm{~S}$. Jang and J. Ih, "On the multiple microphone method for measuring induct acoustic properties in the presence of mean flow," J. Acoust. Soc. Am. 103(3), 1520-1526 (1998).

${ }^{14}$ Y. Aurégan and M. Leroux, "Failures in the discrete models for flow duct with perforations: An experimental investigation," J. Sound Vib. 265, 109-121 (2003).

${ }^{15} \mathrm{U}$. Ingard and H. Ising, "Acoustic nonlinearity of an orifice," J. Acoust. Soc. Am. 42(1), 6-17 (1967).

${ }^{16}$ M. A. Temiz, I. Lopez Arteaga, G. Efraimsson, M. Åbom, and A. Hirschberg, "Acoustic end correction in microperforated platesRevisited," in Proceedings of the 21st International Congress on Sound and Vibration, Beijing, China (2014), pp. 1-8.

${ }^{17} \mathrm{P}$. Morse and K. Ingard, Theoretical Acoustics, 1st ed. (Princeton University Press, Princeton, NJ, 1986), pp. 480-482.

${ }^{18}$ A. D. Pierce, Acoustics: An Introduction to Its Physical Principles and Applications, 2nd ed. (McGraw-Hill, London, 1989), pp. 341-348. 